Session 2630

\title{
Engineering Student Design Processes: Looking at Evaluation Practices Across Problems
}

\author{
Monica E. Cardella ${ }^{1}$, Cynthia J. Atman ${ }^{1,2}$, Robin S. Adams ${ }^{1}$, and Jennifer \\ Turns $^{1,3}$ \\ ${ }^{1}$ Center for Engineering Learning and Teaching/ ${ }^{2}$ Department of Industrial \\ Engineering $/{ }^{3}$ Department of Technical Communication \\ University of Washington, Seattle, Washington
}

\begin{abstract}
The act of evaluating solutions is a common engineering design activity. Over the past eight years we have used verbal protocol analysis to gain insight into engineering students' design processes. This study includes protocols from 32 freshmen and 61 seniors who solved 2 design problems that differed in complexity. In this dataset, 18 of the subjects solved the same problems as both freshmen and seniors. This dataset has allowed us to characterize differences between freshmen and seniors on a global scale as well as an individual scale. Additionally, the inclusion of two problems that vary in complexity allows us to analyze differences in performance and behaviors across problems.
\end{abstract}

One of the important findings that has emerged from an across problem comparison is differences in the amount of time that students spent evaluating their solutions. In particular, $(i)$ students spent more time evaluating their solutions and (ii) a greater number of students evaluated their solutions when solving a more "complex" problem as compared to a less "complex" one. In this paper, we present these results and discuss reasons for these differences. These include differences in the complexity of the two problems and the kinds of processes students employed while designing their solutions. We will also discuss the relationships between time spent evaluating and the number of constraints considered (constraints either given or introduced by the student). We conclude this paper with a summary of implications for engineering education.

Introduction

While engineering disciplines vary in emphasis, a central element to all is design. Some engineers design bridges or structures, some software and other manufacturing tools and assemblies. In addition to job requirements that demand design skills of engineers, current ABET criteria require engineering programs to identify, assess and demonstrate evidence of design competency ${ }^{1}$. In order to satisfy these demands, engineering programs must understand design

"Proceedings of the 2002 American Society for Engineering Education Annual Conference \& Exposition Copyright (C) 2002, American Society for Engineering Education" 
and successful design practices. This can be accomplished by studying the practices of expert designers and investigating students' design behavior. For example, researcher s attempt to identify differences between expert and novice designers-between first year engineering students and graduating engineers as well as between engineering students and practicing engineers.

In previous studies we identified some important differences between freshmen and senior engineering students' design problem solving behaviors ${ }^{2-7}$. One difference is product: in general, freshmen solutions are of lower quality than are the seniors'. However, we have seen that freshmen that spent more time evaluating their solutions tend to generate higher quality solutions than do freshmen who spent little time in evaluation ${ }^{6}$. Time spent evaluating solutions is one of the many observations on students' processes that we have made. We have also noted differences between freshmen and seniors in the total amounts of time spent solving problems and differences in the way freshmen and seniors make transitions between design activities. Examples of these findings are listed below. Our analyses have been summarized in Atman and Turns 5 and Adams, Turns and Atman ${ }^{4}$.

- Time spent

- Time spent in decision step

- Number of transitions

- Transitions rate

- Number of design criteria considered

- Progression to later stages of design process

- Iterative activities

A key element of design studies is the task performed by the study participants. In the current study students solved a series of shorter problems rather than a single, lengthier problem. We have found results similar to our previous studies as well as some differences that illuminate the ways that different design problems elicit different design strategies. In particular, we expect that a more complex design problem will demand more evaluation from the problem solvers than will a less complex problem. In this paper we explore this hypothesis. We begin with a description of our methods and the study tasks, and end with a summary and discussion of our study results.

Method

\section{Subjects}

A total of 75 engineering students from the University of Pittsburgh participated in this study. Initially 32 freshmen engineering students participated; half before beginning their course work and the other 16 just after completing their first semester. Eighteen of the freshmen participated again as seniors as well as an additional 43 seniors.

\section{Procedure}

For this study, engineering students were asked to "think aloud" as they solved a series of openended problems. This study focuses on the first two problems (see Figure 1). Verbal protocol analysis is a well documented method used to identify cognitive processes ${ }^{8}$ and has often been applied to engineering design studies ${ }^{7,9,10}$.

"Proceedings of the 2002 American Society for Engineering Education Annual Conference \& Exposition Copyright (C) 2002, American Society for Engineering Education" 
Figure 1: Problem Statements

\section{PROBLEM ONE: $\quad$ Ping Pong Problem}

In an attempt to avoid boredom at Benedum Hall, creative engineering students developed a challenging new game. A ping-pong ball is to be launched at a bullseye target, and points are awarded according to the accuracy of the landing. However, the ping-pong ball cannot be thrown at the target. It is up to you to design a device which will lift the ping-pong ball into the air and land it at the target. An accurate landing is desired while also maintaining a long flight time. Given that the center of the landing area is 5 meters away from the launch site, and the entire launching assembly must not be greater than $1 \mathrm{~m} \times 1 \mathrm{~m} \times 1 \mathrm{~m}$ in dimension, design a ping-pong ball launcher for this game.

Your work should contain a detailed description of your design and should include any relevant diagrams and calculations. Please clearly state all assumptions which are needed in your analysis and try to keep your design simple yet effective.

\section{PROBLEM TWO: $\quad$ Street Crossing Problem}

College campuses are often overcrowded with pedestrians crossing the streets, since walking is a popular form of transportation for college students. One busy intersection at Pitt is the crossing of Fifth Ave. in front of the bookstore. Dangers at this intersection include heavy traffic and busses which run against the general traffic flow. The University would like to design a cost effective method to cross Fifth Ave. which would reduce the possibility of accidents at this intersection.

Your work should contain a detailed description of your design and should include any relevant diagrams and calculations. Estimate both the costs and the benefits associated with your design. Please clearly state all assumptions which are needed in your analysis and try to keep your design simple yet effective.

To perform verbal protocol analysis, we collected, transcribed, segmented and coded each of the 93 protocols. To enable us to study the students' design processes, two researchers independently coded each transcript with a previously implemented design step coding scheme and then checked for reliability (see Figure 2). If the coders agreed on at least $70 \%$ of the codes they arbitrated by arguing the disagreements until they reached a consensus on all codes. However, if they agreed on less than $70 \%$ of the codes, they recoded the entire transcript. Average inter-rater reliability for the first problem was $80.7 \%$ and $83.3 \%$ for the second problem. To explore the students' design products, we applied a similar process to the scoring of the quality solutions. A team of engineering experts-mechanical and civil engineering professors-developed a scoring rubric which two scorers independently applied to the solutions with an average $93.6 \%$ interrater reliability level. 
Figure 2: Coding Scheme ${ }^{11}$

\begin{tabular}{|c|c|}
\hline Design Step & \\
\hline Identifying a Need & Identify basic needs (purpose, reason for design) \\
\hline Problem definition (PD) & $\begin{array}{l}\text { Define what the problem really is, identify the constraints, identify criteria, reread } \\
\text { problem statement or information sheets, question the problem statement }\end{array}$ \\
\hline Gathering information (GATH) & Search for and collect information \\
\hline Generating ideas (GEN) & Develop possible ideas for a solution, brainstorm, list different alternatives \\
\hline Modelling (MOD) & Describe how to build an idea, measurements, dimensions, calculations \\
\hline Feasibility Analysis (FEAS) & Determine workability, does it meet constraints, criteria, etc. \\
\hline Evaluation (EVAL) & Compare alternatives, judge options, is one better, cheaper, more accurate \\
\hline Decision (DEC) & Select one idea or solution among alternatives \\
\hline Communication (COM) & Communicate the design to others, write down a solution or instructions \\
\hline Implementation & Produce or construct a physical device, product or system \\
\hline
\end{tabular}

Analysis

The segmented transcripts and corresponding codes were entered into MacSHAPA, a software program expressly developed for the analysis of verbal data ${ }^{12}$. This program calculated the amounts of time spent in each design step as well as the total time spent on the problems for each student. Independent samples t-tests were then used to investigate differences between freshmen and seniors. Paired-samples t-tests were used to investigate differences between within-subject performances - that is, the changes in behavior of the freshmen that also solved the problems as seniors. Paired-samples t-tests were also used to investigate differences in performances across the two problems, such as the amount of time each student spent on the first problem compared with the amount of time the student spent on the second problem. To investigate relationships between the quality of the final solution and other design behavior we used Spearman's Rank correlations.

Results

Our results are provided in Tables 1 and 2. Table 1 is a comparison across freshmen and senior participants; Table 2 is a comparison of within-subject data. The majority of the results are consistent with a previous freshmen and senior comparison study ${ }^{6}$. The previous study differs in that the participants solved only one long, open-ended design problem (designing a playground, with the option of asking the experimenter for any needed information) and the study did not include within-subject data. Thus the current study gives us new insight into individual participants' growth-improvement in senior compared to freshmen performances - as well as insight into design process differences attributable to problem type.

For the current study, the seniors continued to create significantly higher quality solutions than did the freshmen (see Table 1). Also consistent with the previous study, the seniors spent a significantly longer amount of time in the evaluation step than did the freshmen. As the definition of the evaluation step suggests, students must generate more than one potential solution in order to make an evaluation. Thus it is not surprising that our results illustrate that in addition to spending more time in evaluation, the seniors also considered more alternative solutions than did the freshmen. These differences were also true for the within-subject

"Proceedings of the 2002 American Society for Engineering Education Annual Conference \& Exposition Copyright (C) 2002, American Society for Engineering Education" 
participants, with the exception of time spent in the evaluation step on the Ping Pong problem (see Table 2).

Table 1: All Participants

\begin{tabular}{|c|c|c|c|c|c|}
\hline & \multicolumn{2}{|c|}{ Ping Pong } & \multicolumn{2}{|c|}{ Street Crossing } \\
\hline & & Freshmen $n=32$ & Seniors $n=61$ & Freshmen $n=32$ & Seniors $n=61$ \\
\hline \multirow[t]{3}{*}{ Quality* } & Mean & 1.02 & 1.46 & 0.32 & 0.48 \\
\hline & Std. Dev. & 0.77 & 0.73 & 0.17 & 0.23 \\
\hline & $p$ value & \multicolumn{2}{|c|}{0.0045} & \multicolumn{2}{|c|}{$<0.0005$} \\
\hline \multirow{3}{*}{$\begin{array}{l}\text { Evaluation } \\
\text { (time in } \\
\text { sec.) }\end{array}$} & Mean & 1.8 & 4.2 & 6.0 & 13.8 \\
\hline & Std. Dev. & 4.2 & 9.6 & 9.0 & 17.4 \\
\hline & $p$ value & \multicolumn{2}{|c|}{0.065} & \multicolumn{2}{|c|}{0.005} \\
\hline \multirow{3}{*}{$\begin{array}{l}\text { Number of } \\
\text { Alternative } \\
\text { Solutions }\end{array}$} & Mean & 1.3750 & 1.3443 & 1.4375 & 2.5082 \\
\hline & Std. Dev. & 0.6599 & 0.6554 & 0.6189 & 1.3244 \\
\hline & $p$ value & \multicolumn{2}{|c|}{0.268} & \multicolumn{2}{|c|}{$<0.0005$} \\
\hline
\end{tabular}

* maximum quality score for Ping Pong is 3.618, for Street Crossing 1.28

Table 2: Within-Subject Participants

\begin{tabular}{|c|c|c|c|c|c|}
\hline & & \multicolumn{2}{|c|}{ Ping Pong } & \multicolumn{2}{|c|}{ Street Crossing } \\
\hline & & Freshmen $n=18$ & Seniors $\mathrm{n}=18$ & Freshmen $\mathrm{n}=18$ & Seniors $n=18$ \\
\hline \multirow[t]{3}{*}{ Quality* } & Mean & 1.05 & 1.85 & 0.31 & 0.54 \\
\hline & Std. Dev. & 0.69 & 0.70 & 0.17 & 0.19 \\
\hline & $p$ value & \multicolumn{2}{|c|}{0.001} & \multicolumn{2}{|c|}{0.001} \\
\hline \multirow{3}{*}{$\begin{array}{l}\text { Evaluation } \\
\text { (time in } \\
\text { min.) }\end{array}$} & Mean & 2.4 & 4.2 & 6.6 & 17.4 \\
\hline & Std. Dev. & 5.4 & 8.4 & 9 & 13.8 \\
\hline & $p$ value & \multicolumn{2}{|c|}{0.233} & \multicolumn{2}{|c|}{0.004} \\
\hline \multirow{3}{*}{$\begin{array}{l}\text { Number of } \\
\text { Alternative } \\
\text { Solutions }\end{array}$} & Mean & 1.5000 & 1.3330 & 1.3889 & 2.4440 \\
\hline & Std. Dev. & 0.7859 & 0.6859 & 0.6077 & 1.0970 \\
\hline & $p$ value & \multicolumn{2}{|c|}{0.235} & \multicolumn{2}{|c|}{0.003} \\
\hline
\end{tabular}

* maximum quality score for Ping Pong is 3.618, for Street Crossing 1.28

The majority of these results are consistent across problems. However, there were some notable differences. First, the allocation of time in evaluation practices differed considerably. A greater number of students spent time in the evaluation step on the Street Crossing problem than on the Ping Pong problem-62 compared to 22 . Additionally, all of the students spent significantly more time in evaluation on the Street Crossing problem (mean $=11.4 \mathrm{sec})$ than on the Ping Pong problem $($ mean $=1.8 \mathrm{sec}), p<0.005$.

"Proceedings of the 2002 American Society for Engineering Education Annual Conference \& Exposition Copyright (C) 2002, American Society for Engineering Education" 


\section{Discussion}

The Ping Pong and Street Crossing problems used in the current study differ in complexity and in the participants' familiarity with the context. The first, which asks students to design a ping pong ball launcher, is similar to a typical engineering homework problem. In fact, one student commented during the task on having solved a similar problem in class. The second problem, which asks students to devise a means for safely crossing a busy street, is more context specific - the problem's street is one that students frequently crossed to get to school.

Additionally, the students are asked to perform more tasks during this problem. Specifically, they are asked to estimate the costs and benefits of their solutions in addition to simply designing a viable solution. These additional components of the problem, which increase the complexity of the problem, may be related to the more prevalent evaluation behavior. Other studies illuminate some of the relationships between constraints considered and evaluation.

In particular, from our previous study ${ }^{6}$ for which participants designed a playground, we learned that students who gathered more information spent more time in evaluation. For both the freshmen and the seniors, the amount of time spent in the evaluation step strongly and positively correlated with the number of assumptions made. For example, some students made assumptions about the size of children when they calculated equipment heights and weight requirements rather than asking for this information. In addition to these implicit information requests, the total number of explicit information requests strongly correlated with the amount of time in evaluation for both the freshmen and the seniors. While few other correlations with evaluation are noteworthy for the seniors, for the freshmen the total number of information categories covered also correlated with more time spent in evaluation. This means that the greater the number of different types of information that the freshmen covered, the more evaluation they performed. Overall, implicit and explicit information gathering activities were correlated with time spent in evaluation activities. This means that the students who sought more information also tended to spend more time evaluating their solutions. This added information translates into what Ullman, Dietrich and Staufer ${ }^{13}$ might call more "introduced" constraints.

Ullman, Dietrich and Staufer ${ }^{13}$ suggest three types of constraints in their model of the mechanical engineering design process. Given constraints emerge directly from the problem statement. In the case of the Street Crossing problem, given constraints are that the design must reduce accidents and improve safety. Introduced constraints come from the designer's domain knowledge. While $9 \%$ of the current study's participants drew on personal experience in solving the Ping Pong problem, 22\% did so in the Street Crossing problem. For example, students remembered positions of buildings near the problem's street that constrained the problem. Because the Street Crossing problem was more context specific, it is not surprising that students drew on more domain knowledge for this problem. Ullman, Dietrich and Staufer ${ }^{13}$ call the third type derived constraints. These constraints are derived from the potential solutions that the designer envisions. On the Street Crossing problem, the participants thought of more solution possibilities than they did for the Ping Pong problem. This difference is significant for the group of all participants, $p<0.0005$, and for the group of all seniors, $p<0.0005$, but not the group of only freshmen, $p=0.721$ (see Table 3 ).

"Proceedings of the 2002 American Society for Engineering Education Annual Conference \& Exposition Copyright (C) 2002, American Society for Engineering Education" 
Table 3: Alternative Solution Types

\begin{tabular}{|c|c|c|c|}
\hline & & Ping Pong & Street Crossing \\
\hline \multirow{3}{*}{$\begin{array}{c}\text { All } \\
n=93\end{array}$} & Mean & 1.3548 & 2.1398 \\
\hline & Std. Dev. & 0.6535 & 1.2388 \\
\hline & $p$ value & \multicolumn{2}{|c|}{$<0.0005$} \\
\hline \multirow{3}{*}{$\begin{array}{c}\text { Seniors } \\
n=61\end{array}$} & Mean & 1.3443 & 2.5082 \\
\hline & Std. Dev. & 0.6554 & 1.3244 \\
\hline & $p$ value & \multicolumn{2}{|c|}{$<0.0005$} \\
\hline \multirow{3}{*}{$\begin{array}{c}\text { Freshmen } \\
\mathrm{n}=32\end{array}$} & Mean & 1.3750 & 1.4375 \\
\hline & Std. Dev. & 0.6599 & 0.6189 \\
\hline & $p$ value & \multicolumn{2}{|c|}{0.721} \\
\hline
\end{tabular}

For each of the three types of constraints, there is a tendency for more evaluation behavior to occur in the scenario when there are more constraints to attend to. Thus it is likely that the more constraint-laden nature of the Street Crossing problem induced more evaluation behavior. However, future work is necessary to investigate a causal relationship rather than a correlation between constraints and evaluation.

The previous study, involving the design of a playground, suggested that investigation is worthwhile because of the relationship between evaluation and the quality of the final solution. The current study supports and extends this finding. The students who spent time in evaluation on the Street Crossing problem generated solutions of significantly higher quality. This expands our previous finding - that freshmen that spent time in evaluation produced higher quality solutions - to a larger set of participants. It also echoes the difference in problems; evaluation did not play such a crucial role in the more standard problem but it did in the more complex and context-specific problem.

\section{Conclusion}

The noticeably different role of evaluation on problems that differ in complexity suggests two implications for future research. First, the choice of task has an impact on problem solving behavior. Our findings indicate that the participants in this study identified fewer constraints and thus spent less time in evaluation on a less complex, more homework-like problem. In comparison, the Street Crossing problem elicited more evaluation. This is probably due to more constraint identification and the additional familiarity with the context of the problem. From this we conclude that design researchers must carefully choose the study task(s). More importantly, design educators must carefully choose the problems that they ask their students to solve if they want to encourage a broader exposure to important design activities.

The fact that the students exhibited significantly more evaluation behaviors for the Street Crossing problem than the Ping Pong problem suggests that students should be exposed to a wide variety of problems as part of their engineering education in order to practice all the skills

"Proceedings of the 2002 American Society for Engineering Education Annual Conference \& Exposition Copyright (C) 2002, American Society for Engineering Education" 
necessary to become competent practitioners ${ }^{14}$. In this case, the students benefited from the problem's prompts to perform more tasks and consider more introduced constraints as this may have enabled them to spend more time making evaluations. Ultimately, however, students need to learn to consider these constraints in a prompt-free environment. While these results may not be surprising to educators, they provide an empirical basis that can be used to inform design education.

\section{Acknowledgments}

This work was supported in part by grants from the GE Fund and NSF grants DUE-9254271, RED-9358516, DGE-9714459 and EEC-9872498. We would like to gratefully acknowledge the students who participated in the study, and the students who helped us to code the protocols, specifically Jacob Burghardt, Louise Cheung, Jennifer Chin, Julie Christianson, Cathie Scott, Jennifer Temple and Bettina Vuong.

\section{References}

1 Accreditation Board for Engineering and Technology (ABET) (1998). Engineering Criteria 2000: Criteria for accrediting programs in engineering in the United States (2nd edition ed.). Baltimore, Engineering Accreditation Commission, Accreditation Board for Engineering and Technology.

2. Adams, Robin S. (2001). Cognitive processes in iterative design behavior. Dissertation: University of Washington.

3. Adams, Robin S. and Cynthia J. Atman (1999). Characterizing Engineering Student Design Processes: An Illustration of Iteration. Proceedings of the Annual Conference for the American Society of Engineering Education, Charlotte, NC.

4. Adams, Robin, Jennifer Turns and Cynthia J. Atman, (2001). Educating Effective Engineering Designers: The Role of Reflective Practice. Proceedings of the 2001 Design Thinking Research Symposium 5, Delft University of Technology, Netherlands, December.

5. Atman, Cynthia J. and Jennifer Turns (2001). Studying Engineering Design Learning: Four Verbal Protocol Analysis Studies. Design Learning and Knowing. M. McCracken, Newstetter, W., and Eastman, C. New Jersey, Lawrence Erlbaum.

6. Atman, Cynthia J., Justin R. Chimka, Karen M. Bursic, and Heather L. Nachtmann (1999), "A comparison of freshman and senior engineering design processes," Design Studies 20 (2), 131-152.

7. Mullins, Carie A., Cynthia J. Atman, and Larry J. Shuman (1999), “Freshmen Engineers' Performance When Solving Design Problems," IEEE Transactions on Education, 42 (4), 281-287.

8. Ericsson, K.A. and H. A. Simon (1984). Protocol Analysis: verbal reports as data. Cambridge, The MIT Press.

9. Atman, Cynthia J. and Karen M. Bursic (1998), “Verbal Protocol Analysis as a Method to Document engineering Student Design Processes,” Journal of Engineering Education 87 (2), 121-132.

10. Gero, John S. and Thomas Mc Neill (1998), "An approach to the analysis of design protocols," Design Studies 19 (1), 21-61.

"Proceedings of the 2002 American Society for Engineering Education Annual Conference \& Exposition Copyright (C) 2002, American Society for Engineering Education" 
11. Atman, Cynthia J. and Karen M. Bursic (1996). Teaching Engineering Design: Can Reading a Textbook Make a Difference? Research in Engineering Design 7(7): 240-250.

12. Sanderson, P.M., J.J.P. Scott, T. Johnston, J. Mainzer, L.M.Watanabe, and J.M. James (1994), "MacSHAPA and the enterprise of Exploratory Sequential Data Analysis (ESDA)", International Journal of Human-Computer Studies, 41 (5), 633-681.

13. Ullman, David G. , Thomas G. Dietterich and Larry A. Staufer (1988), "A Model of Mechanical Design Process Based on Empirical Data," AI in Engineering Design and Manufacturing, 2(1), 33-52.

14. Woods, D.R. (1995), "Problem-based Learning: Helping your students gain the most from PBL" D.R. Woods Publishing, Waterdown, ON.

"Proceedings of the 2002 American Society for Engineering Education Annual Conference \& Exposition Copyright (C) 2002, American Society for Engineering Education", 